\title{
On the Nature of Exotic Matter in Morris-Thorne Wormholes
}

\author{
Peter K F Kuhfittig \\ Department of Mathematics, Milwaukee School of Engineering, Milwaukee, Wisconsin 53202-3109, USA \\ Email: kuhfitti@msoe.edu
}

\begin{abstract}
It is well known that phantom energy, which is characterized by the equation of state $p=\omega \rho, \omega<-1$, can support Morris-Thorne wormholes since it leads to a violation of the null energy condition. The purpose of this note is to show that the converse is also true in the following sense: for a typical shape function, the equation of state of exotic matter in the vicinity of the throat is given by $p_{r}=\omega \rho, \omega<-1$, where $p_{r}$ is the radial pressure.
\end{abstract}

Keywords: Morris-Thorne wormholes, exotic matter, phantom energy

\section{Introduction}

Wormholes are tunnel-like structures in spacetime that connect widely separated regions of our Universe or different universes in a multiverse. Apart from some forerunners, macroscopic traversable wormholes were first studied in detail by Morris and Thorne [1] in 1988. They had proposed the following static and spherically symmetric line element for a wormhole spacetime:

$$
d s^{2}=-e^{2 \Phi(r)} d t^{2}+\frac{d r^{2}}{1-b(r) / r}+r^{2}\left(d \theta^{2}+\sin ^{2} \theta d \phi^{2}\right)
$$

using units in which $c=G=1$. Here $\Phi=\Phi(r)$ is called the redshift function, which must be everywhere finite to prevent an event horizon. The function $b=b(r)$ is called the shape function since it determines the spatial shape of the wormhole when viewed, for example, in an embedding diagram [1]. The spherical surface $r=r_{0}$ is called the throat of the wormhole, where $b\left(r_{0}\right)=r_{0}$. The shape function must also meet the requirement $b^{\prime}\left(r_{0}\right)<1$, called the flare-out condition, while $b(r)<r$ for $r>r_{0}$. We also require that $b^{\prime}\left(r_{0}\right)>0$.

The flare-out condition can only be met by violating the null energy condition (NEC) which states that

$$
T_{\alpha \beta} k^{\alpha} k^{\beta} \geq 0
$$

for all null vectors $k^{\alpha}$, where $T_{\alpha \beta}$ is the energy-momentum tensor. Matter that violates the NEC is called "exotic" in Ref. [1]. Exotic matter is usually confined to a narrow region around the throat. Now observe that for the outgoing null vector $(1,1,0,0)$, we have

$$
T_{\alpha \beta} k^{\alpha} k^{\beta}=\rho+p_{r}<0 ;
$$

so the NEC has been violated. Here $T_{t}^{t}=-\rho$ is the energy density, $T_{r}^{r}=p_{r}$ is the radial pressure, and $T_{\theta}^{\theta}=T_{\phi}^{\phi}=p_{t}$ is the lateral (transverse) pressure. Before continuing, let us list the Einstein field equations:

$$
\begin{gathered}
\rho(r)=\frac{b^{\prime}}{8 \pi r^{2}}, \\
p_{r}(r)=\frac{1}{8 \pi}\left[-\frac{b}{r^{3}}+2\left(1-\frac{b}{r}\right) \frac{\Phi^{\prime}}{r}\right], \\
p_{t}(r)=\frac{1}{8 \pi}\left(1-\frac{b}{r}\right)\left[\Phi^{\prime \prime}-\frac{b^{\prime} r-b}{2 r(r-b)} \Phi^{\prime}+\left(\Phi^{\prime}\right)^{2}+\frac{\Phi^{\prime}}{r}-\frac{b^{\prime} r-b}{2 r^{2}(r-b)}\right] .
\end{gathered}
$$

Since Eq. (6) can be obtained from the conservation of the stress-energy tensor $T_{; \nu}^{\mu \nu}=0$, only Eqs. (4) and (5) are actually needed. 
The discussion of exotic matter took a dramatic turn with the discovery that the Universe is expanding at an accelerated rate caused by a hypothesized dark energy $[2,3]$, which makes up about $70 \%$ of the substance in the Universe. Dark energy is characterized by its barotropic equation of state $p=\omega \rho$, where $\rho>0$ and $\omega<-1 / 3$. The range $\omega<-1$, called phantom energy, is of particular interest to us since it leads to a violation of the NEC $[4,5]$ :

$$
p+\rho=\omega \rho+\rho=(\omega+1) \rho<0 .
$$

To apply the equation of state $p=\omega \rho$ to wormholes, where the pressure is generally anisotropic, one regards the pressure in the equation of state as a negative radial pressure, and the tangential pressure is obtained from the Einstein field equations. Moreover, according to Ref. [6], moving away from the throat, the radial and transverse pressures rapidly become equal, showing that phantom energy can in principle support traversable wormholes.

The main purpose of this note is to obtain a converse in the following sense: for any Morris-Thorne wormhole with a typical shape function, the exotic matter near the throat has the equation of state $p_{r}=\omega \rho, \omega<-1$.

\section{A Generic Shape Function}

Consider the family of functions [1]:

$$
b_{\eta}(r)=r_{0}\left(\frac{r}{r_{0}}\right)^{1-\eta}, \quad 0<\eta<1 .
$$

Evidently, $b_{\eta}\left(r_{0}\right)=r_{0}$, while

$$
0<b_{\eta}^{\prime}\left(r_{0}\right)=1-\eta<1
$$

thereby meeting the basic requirements of a shape function. The special case $\eta=1 / 2$ leads to the parabola $b(r)=\sqrt{r_{0} r}$, which is concave down to the right of $r=r_{0}$ with $b(r)<r$ for all $r$. The same behavior is exhibited by all members of the family in Eq. (8). Moreover, for every value of $m$ between 0 and 1 , there exists a member $b_{\eta}(r)$ such that $b_{\eta}^{\prime}\left(r_{0}\right)=m$.

These general properties are enough to suggest that any shape function of a Morris-Thorne wormhole can be closely approximated by a member of $\left\{b_{\eta}(r)\right\}$, at least in the vicinity of the throat. To that end, we need to assume that a typical shape function $b=b(r)$ is concave down in the immediate vicinity of $r=r_{0}$ with $b(r)<r$. In other words, $b(r)$ has the following properties:

I. $b\left(r_{0}\right)=r_{0}$, where $r=r_{0}$ is the throat of the wormhole,

II. $0<b^{\prime}\left(r_{0}\right)<1$,

III. $b(r)$ is concave down for $r$ near $r=r_{0}$.

Property I is automatically satisfied by $b_{\eta}(r)$. Regarding Property II, the value of $b^{\prime}\left(r_{0}\right)$ determines $\eta$ by Eq. (9). Finally, for any $\eta \varepsilon(0,1)$,

$$
b^{\prime \prime}\left(r_{0}\right)=\frac{1}{r_{0}}(1-\eta)(-\eta)<0
$$

so that $b_{\eta}(r)$ is indeed concave down near $r=r_{0}$ (Property III). As a result, any shape function that meets Conditions I, II, and III can be approximated by some $b_{\eta}(r)$ in the vicinity of the throat.

\section{The Equation of State}

By making use of the properties of $b=b(r)$ discussed in Sec. 2, we are going to obtain the equation of state for exotic matter in the vicinity of the throat. As already noted, this is precisely the region where we would expect to find exotic matter. First we observe that in Eq. (5), the second term inside the brackets can be neglected. We now obtain

$$
\rho(r)=\frac{1}{8 \pi} \frac{1}{r^{2}}(1-\eta)\left(\frac{r_{0}}{r}\right)^{\eta}
$$


and

$$
p_{r}(r)=\frac{1}{8 \pi}\left(-\frac{1}{r^{3}}\right)\left(r_{0}\right)\left(\frac{r}{r_{0}}\right)^{1-\eta} .
$$

Eqs. (10) and (11) lead to the surprising result that $\rho(r) / p_{r}(r)$ reduces to a constant value for all $r$ :

$$
\frac{\rho(r)}{p_{r}(r)}=\eta-1 \quad \text { or } \quad p_{r}(r)=\frac{1}{\eta-1} \rho(r) .
$$

If we now write

$$
p_{r}(r)=\omega \rho(r),
$$

we conclude that, since $0<\eta<1$,

$$
\omega<-1 .
$$

So Eq. (13) has the same form as the equation of state for phantom energy, to be discussed further in the next section. Our main conclusion is that the unavoidable exotic matter not only violates the null energy condition, it can be characterized by the equation of state (13), where $\omega<-1$.

\section{Returning to Phantom Energy}

While Eq. (13) has the same form as the cosmological equation of state $p(r)=\omega \rho(r)$ for phantom energy, there is one critical difference. For the latter, both $p$ and $\rho$ are necessarily small. For the former, $p_{r}(r)=\omega \rho(r)$, the opposite is true, as already pointed out in Ref. [1]: recalling that the tension $\tau(r)$ is the negative of $p(r)$, we can rewrite Eq. (5) as (reintroducing $c$ and $G$ for now)

$$
\tau(r)=\frac{b(r) / r-2[r-b(r)] \Phi^{\prime}(r)}{8 \pi G c^{-4} r^{2}} .
$$

According to Ref. [1], the radial tension at the throat is

$$
\tau=\frac{1}{8 \pi G c^{-4} r_{0}^{2}} \approx 5 \times 10^{41} \frac{\mathrm{dyn}}{\mathrm{cm}^{2}}\left(\frac{10 \mathrm{~m}}{r_{0}}\right)^{2} .
$$

In particular, for $r_{0}=3 \mathrm{~km}, \tau$ has the same magnitude as the pressure at the center of a massive neutron star. For a phantom-energy wormhole, such an outcome is clearly absurd: for such a wormhole to exist, $r=r_{0}$ would have to be extremely large in view of Eq. (15). So phantom-energy wormholes could only exist on very large scales.

Remark: It is proposed in Ref. [7] that the enormous radial tension can be attributed to (1) the effect of noncommutative geometry, an offshoot of string theory, or (2) the existence of a small extra spatial dimension.

\section{Conclusion}

The unavoidable exotic matter in a Morris-Thorne wormhole is assumed to be confined to the immediate vicinity of the throat. In this note we also assume that a typical shape function is concave down near $r=r_{0}$ (in addition to its usual properties). It is subsequently shown that the equation of state of the exotic matter is $p_{r}=\omega \rho, \omega<-1$, which is essentially the same as the equation of state for phantom dark energy, since, in a phantom-energy wormhole, the radial and lateral pressures tend to quickly become equal away from the throat [6]. So while phantom energy behaves like exotic matter, the converse is also true since the equation of state of exotic matter has the same form. There is one essential difference, however: since $p$ and $\rho$ are necessarily small in the phantom-energy case, it follows from Eq. (15) that such wormholes could only exist on very large scales. 


\section{References}

1. M.S. Morris and K.S. Thorne, "Wormholes in spacetime and their use for interstellar travel: A tool for teaching general relativity," Amer. J. Phys. 56, pp. 395-412 (1988).

2. A.G. Riess et al., "Observational evidence from supernovae for an accelerating universe and a cosmological constant," Astron. J. 116, pp. 1009-1038 (1988).

3. S. Perlmutter, M.S. Turner and M. White, "Constraining dark energy with Type 1a supernovae and large-scale structure," Phys. Rev. Lett. 83, pp. 670-673 (1999).

4. S. Sushkov, "Wormholes supported by a phantom energy," Phys. Rev. D 71, ID: 043520 (2005).

5. F.S.N. Lobo, "Phantom energy traversable wormholes," Phys. Rev. D 71, ID: 084011 (2005).

6. S.V. Sushkov and S.-W. Kim, "Cosmological evolution of a ghost scalar field," Gen. Rel. Grav. 36, pp. 1671-1678 (2004).

7. P.K.F. Kuhfittig, "Accounting for the large radial tension in Morris-Thorne wormholes," Eur. Phys. J. Plus 135, ID: 510 (2020). 